\title{
Patients with acute poisoning presenting to an urban emergency department of a tertiary hospital in Tanzania
}

\author{
Ghaniya S. Mbarouk2 ${ }^{2}$ Hendry R. Sawe ${ }^{1,2^{*}}$, Juma A. Mfinanga ${ }^{1,2}$, John Stein ${ }^{3}$, Shankar Levin ${ }^{3}$, Victor Mwafongo ${ }^{1,2}$, \\ Michael S. Runyon ${ }^{1,4}$, Teri A. Reynolds ${ }^{1,3,5}$ and Kent R. Olson ${ }^{6}$
}

\begin{abstract}
Background: Poisoning is a major public health concern in sub-Saharan Africa, affecting patients of all age groups. Poisoned patients often present to the emergency department (ED) and prompt evaluation and appropriate management are imperative to ensure optimal outcomes. Unfortunately, little is known about the specific presentations of poisoned patients in East Africa. We describe the clinical and epidemiological features of patients presenting to the Muhimbili National Hospital (MNH) ED with suspected toxicological syndromes.

Methods: This prospective study enrolled a consecutive sample of ED patients who presented with a suspected toxicological syndrome from March 2013 to June 2013. Trained investigators completed a structured case report form (CRF) for each eligible patient, documenting the suspected poison, demographic information, the clinical presentation, and the ED outcome and disposition. The study data were analyzed and summarized with descriptive statistics.
\end{abstract}

Results: Of 8827 patients, who presented to ED-MNH, 106 (1.2\%) met inclusion criteria, and all were enrolled. Among those enrolled, the median age was 28 years (interquartile range [IQR] 16 years), and 81 (76.4\%) were male. Overall 55 (52\%) were single, and 28 (26.4\%) had professional jobs. 60 (56.6\%) patients were referred from district hospitals, 86.8\% of which were in Dar es Salaam. Only 13 (12.3\%) of patients presented to the ED within $2 \mathrm{~h}$ of the toxic exposure. The etiology of poisoning included alcohol in $42(50 \%)$, a mixture of different medications in $12(14.3 \%)$, and snakebite in $6(11.3 \%)$. Most exposures were intentional (63 [59.4\%]) and were via the oral route (88 [83\%]). The most common abnormal physical findings were altered mental status (66 [62.3\%]) and tachypnoea (68 [64.2\%]). One patient died in the ED and 98 (92.5\%) required hospital admission.

Conclusions: Most patients presenting to the ED with a toxicological syndrome were adult males with intentional exposures. The most common toxic exposure was alcohol (ethanol) intoxication and the most common abnormal findings were altered mental status and tachypnoea. More than three-quarter of patients presented after $2 \mathrm{~h}$ of exposure. Almost all patients were admitted to the hospital.

Keywords: Toxicology, Poisoning in Africa, Poisoning diagnosis, ED in Africa

\section{Background}

Acute poisoning is a major medical emergency that carries significant morbidity and mortality in patients of all age groups across the world [1-4]. The World Health

\footnotetext{
*Correspondence: hendry_sawe@yahoo.com

1 Emergency Medicine Department, Muhimbili University of Health and Allied Sciences, Dar es Salaam, P.O. Box 54235, Tanzania

Full list of author information is available at the end of the article
}

Organization (WHO) estimates that poisoning results in an annual loss of 7.4 million years of healthy life (disability adjusted life years) globally. However, this burden disproportionally impacts low and middle-income countries, where over ninety percent of deaths due to unintentional poisoning occur [5].

Acute poisoning may be intentional or unintentional, and studies have shown that intentional poisoning is more common in adults and accidental poisoning is more 
common in children [6]. Patients with acute poisoning have highly variable clinical presentations, making diagnosis difficult, especially in resource limited settings $[7,8]$. The variability of the clinical presentation is further compounded by the fact that substances involved in poisonings vary by age group, intention, geographic region, and level of economic development [6, 9-11]. In the United States, the American Association of Poison Control Centers reports that the top five substance classes most frequently involved in human exposures are analgesics, cosmetics, household cleaning substances, sedatives/hypnotics/antipsychotics, and foreign bodies [8]. European data indicates a different pattern, reflective of the fact that most poisoned patients are adults with suicidal intention, with illicit drugs (mostly benzodiazepines), sedatives/hypnotics/antipsychotics, alcohol, and carbon monoxide as the most common exposures [10].

In Sub Saharan Africa, there is a general paucity of Emergency Department (ED) data describing patterns of acute poisoning, with only a few hospital and mortuary based studies [12-14]. In Tanzania, ED based studies reporting on acute poisoning are non-existent, but poisonings have been documented in other clinical settings. In one study of suicides conducted in the mortuary of the main tertiary referral hospital, poisoning was the most common method suicide, mainly from ingesting antimalarials and pesticides [15]. Another autopsy based surveillance study conducted in southern Tanzania documented 300 accidental cyanide poisoning deaths from consumption of bitter cassava during a country-wide drought in 2002-2003 [16]. Poisonings, have also been documented in mining operations (occupational exposures) and among affected pregnant women presenting to a tertiary referral hospital [17-19].

In acute settings, early recognition and appropriate management have been known to improve outcomes of poisoned patients [20]. Delayed recognition and suboptimal management may lead to increased morbidity and mortality [21]. The case fatality rates vary by poison and region. In Africa and Asia, fatalities have been shown to be highest from pesticides, followed by medications and household products, while paracetamol was found to be a significant cause of poisoning in other regions of the developing world [6].

In high resource settings, poison control centres are common [22] and most emergency physicians managing poisoned patients have the benefit of real time consultation with the expert toxicologists staffing those centres. Thus, in most EDs in the developed world, physicians are able quickly identify the type of poisoning and institute appropriate management, increasing the likelihood of satisfactory outcomes [23]. Unfortunately, there are currently no formal Poison Control Centres or toxicological consultation services readily available to providers in most Sub Saharan Africa countries, including Tanzania. Recognizing toxic cases is still a challenge in Tanzania because there are relatively few acute care providers with the training necessary to detect the subtle and variable presentations of toxicological syndromes. This issue is compounded by lack of access to laboratory toxicological analysis.

Therefore, we aimed to describe the population of patients presenting to the ED of a large public tertiary care hospital with suspected acute poisoning or other toxic exposure, characterizing their presenting signs and symptoms, and reporting on suspected toxicological agents involved. This was hoped to increase providers' knowledge of patients with suspected toxicological syndromes presenting to EDs and other acute intake areas in Tanzania, thereby informing the development of diagnostic and management protocols and guiding educational and clinical initiatives to reduce the morbidity and mortality from toxicological disease.

\section{Methods \\ Study design}

This was a prospective observational study of a consecutive sample of patients presenting to the MNH-ED from 15th March 2013 to 17th June 2013.

\section{Study setting and population}

MNH is a tertiary referral hospital in Dar es Salaam, Tanzania and the main clinical training site for the Muhimbili University of Health and Allied Sciences (MUHAS). The ED was opened in 2010, and it is the site for the only emergency medicine residency program in the country. The MNH-ED receives high acuity patients from within Dar es Salaam and the surrounding regional and district hospitals and served 50,000 patients in 2013. The top five patient presentations are trauma, infectious disease, mental health, neoplasm and pregnancy related complications [24].

The department is staffed by locally trained emergency physicians, who provide clinical care, supervision and teaching to interns (fresh graduates from medical school), registrars (generalists) and emergency medicine residents.

In this study, all patients presenting with suspected poisoning were eligible for enrollment. Suspicion of poisoning was determined by the treating physician based on the history, suggestive clinical picture or presence of the containers or wrappers of the poisonous agent or medications. 


\section{Study protocol}

\section{Operational definition}

Toxicological syndrome was defined as a group of signs and symptoms constituting the basis for a diagnosis of poisoning.

\section{Inclusion criteria}

All patients presenting to $\mathrm{MNH}$ ED with provider impressions including but not limited to: alcohol intoxication, recreational or prescription drug overdose, toxic inhalation, chemical skin exposure, accidental or intentional poisoning, and those who had a snakebite or envenomation.

The treating physicians screened and enrolled patients consecutively with support monitoring by one of the study authors (GM). Data collection was done 24 h everyday and 7 days per week. Providers obtained informed consent from all eligible patients or their accompanying eligible proxy. A standardized case report form (CRF) with explicitly defined variables was used to prospectively collect the study data, including age, sex, occupation and other demographic data, as well as substance(s) suspected, details of exposure, prior psychiatric history, household medications, clinical presentation, ED outcomes and disposition.

\section{Data analysis}

The study data recorded in the CRF were transferred into an Excel database (Microsoft corporation, Redmond, WA), cleaned, and analyzed with Excel and Stata (version 13, StataCorp LP, Texas, USA). Continuous variables were summarized as means and standard deviations (SD) or medians and interquartile ranges (IQR), depending upon the data distribution. Categorical variables are summarized as counts and percentages.

\section{Results}

\section{Study population demographics and referral status} Out of 8827 patients who presented to ED-MNH, 106 (1.2\%) met inclusion criteria and all were enrolled. The majority (81 [76.4\%]) were male and over the age of 18 years, (84 [79.2\%]). The overall median age was 28 years (interquartile range [IQR] 16 years; range 1 month to 91 years) (Table 1 ). Most patients were single, and nearly one-third had a primary level of education or less.

Geographical location of exposure, referral status and time of arrival

Most toxic exposures occurred within the Dar es Salaam region (92 [86.8\%]), with the highest rate in the Kinondoni district (35 [38.0\%]) compared to the Ilala (29 [31.5\%]) and Temeke (18 [19.6\%]) districts (all of which
Table 1 Demographic characteristics of the patients

\begin{tabular}{|c|c|}
\hline Age and gender & $N=106$ \\
\hline \multicolumn{2}{|l|}{ Age } \\
\hline Median (IQR) & 28 (16) years \\
\hline \multicolumn{2}{|l|}{ Age groups (years) } \\
\hline$<5$ & $11(10.4 \%)$ \\
\hline 5 to $<18$ & $11(10.4 \%)$ \\
\hline$\geq 18$ & $84(79.2 \%)$ \\
\hline \multicolumn{2}{|l|}{ Gender } \\
\hline Male & $81(76.4 \%)$ \\
\hline Female & $25(23.6 \%)$ \\
\hline \multicolumn{2}{|l|}{ Marital status } \\
\hline Single & $55(51.9 \%)$ \\
\hline Married & $35(33.0 \%)$ \\
\hline Others $^{a}$ & $3(2.8 \%)$ \\
\hline Unknown & $13(12.3 \%)$ \\
\hline \multicolumn{2}{|l|}{ Level of education } \\
\hline Primary school & $34(32.1 \%)$ \\
\hline Secondary school & $25(23.6 \%)$ \\
\hline College and University & $17(16.0 \%)$ \\
\hline None & $13(12.3 \%)$ \\
\hline Unknown & $17(16.0 \%)$ \\
\hline \multicolumn{2}{|l|}{ Occupation } \\
\hline Professional job $b^{b}$ & $28(26.4 \%)$ \\
\hline Unemployed & $18(17 \%)$ \\
\hline Student & $17(16 \%)$ \\
\hline Others $^{c}$ & $11(10.4 \%)$ \\
\hline Driver & $10(9.4 \%)$ \\
\hline Guard & $10(9.4 \%)$ \\
\hline Farmer & $2(1.9 \%)$ \\
\hline Unknown & $10(9.4 \%)$ \\
\hline \multicolumn{2}{|c|}{ Includes divorced, widowed } \\
\hline \multicolumn{2}{|c|}{$\begin{array}{l}\text { b Nurse, Engineer, Lawyer, Businessman, Secret service, Machine operator, } \\
\text { Secretary, Surveyor, Veterinary doctor, Social worker, Accountant }\end{array}$} \\
\hline
\end{tabular}

are within Dar es Salaam), Most exposures occurred at home $[41(38.7 \%)]$ or recreational places (36 [34.0\%]) (Table 2).

Just over one half of patients were referred from district hospitals, and one quarter presented directly to the MNH-EMD.

Only $13(12.3 \%)$ presented to the MNH-EMD within $2 \mathrm{~h}$ after the toxic exposure. The largest group (42 [39.6\%]) presented within $2-12 \mathrm{~h}$ followed by (32 $[30.2 \%]$ ) who presented after more than $12 \mathrm{~h}$ (Table 2 ).

Patients' clinical presentation and laboratory result Most patients had no psychiatry history (90 [84.9\%]) and were not on chronic medications (92 [86.8\%]). Most patients presented with normal temperatures (101 
Table 2 Geographical location of exposure, referral status and time of arrival

\begin{tabular}{|c|c|}
\hline \multicolumn{2}{|l|}{ Region } \\
\hline Dar es Salaam & $92(86.8 \%)$ \\
\hline Coastal & $9(8.5 \%)$ \\
\hline Other regions $^{\mathrm{a}}$ & $5(4.7 \%)$ \\
\hline \multicolumn{2}{|l|}{ Place } \\
\hline Home & $41(38.7 \%)$ \\
\hline Recreational area & $36(34.0 \%)$ \\
\hline At work & $10(9.4 \%)$ \\
\hline Farm & $1(0.9 \%)$ \\
\hline Others $^{\mathrm{b}}$ & $18(17.0 \%)$ \\
\hline \multicolumn{2}{|l|}{ Referral status } \\
\hline District hospital & $60(56.6 \%)$ \\
\hline Self referral & $26(24.5 \%)$ \\
\hline Brought by police & $13(12.3 \%)$ \\
\hline Others ${ }^{c}$ & $7(6.6 \%)$ \\
\hline \multicolumn{2}{|c|}{ Presenting time after exposure } \\
\hline$<2 \mathrm{~h}$ & $13(12.3 \%)$ \\
\hline $2-12 \mathrm{~h}$ & $42(39.6 \%)$ \\
\hline$>12 \mathrm{~h}$ & $32(30.2 \%)$ \\
\hline Unknown exposure time & $19(17.9 \%)$ \\
\hline
\end{tabular}

[95.3\%]), normal random blood glucose (92 [86.8\%]), normal-sized pupils (83 [78.3\%]), normal skin findings (78 [73.6\%]) and normal pulse rate (69 [65.1\%]). The most common abnormal findings included tachypnoea (68 [64.2\%]) and altered mental status (66 [62.3\%]) (Table 3). A small number of patients 7 (6.6\%) had a mixture of symptoms, including abdominal cramps, swollen lower limb, excessive salivation, convulsions, urinary retention, bloody secretions, neck and jaw stiffness and lethargy.

\section{Suspected agents for all age groups}

The top two toxic agents encountered by adult patient were alcohol (42 [50\%]) taken alone, and mixtures of different medications (12 [14.3\%]), including alcohol plus sedatives, ethanol plus rat poisoning, ethanol plus antimalarial and antibiotics, ethanol plus unknown agents. No specific agent was identified in 8 (9.5\%) of adult patients.

In children below the age of 18 years top three toxic agents were snake venom (6 [27.3\%]), kerosene (3 [13.6\%]) and rat poison (3 [13.6\%]) (Table 4).

\section{Route of exposure and intent for all age groups}

Overall, nearly 63 (59.4\%) of the toxic exposures were intentional, and intentional poisoning was most common
Table 3 Patients' clinical presentation and laboratory results

\begin{tabular}{llll}
\hline Characteristic & Findings & & \\
\hline Vital signs & Normal & Low & High \\
Temperature $(\mathrm{N}=106)$ & $101(95.3 \%)$ & 0 & $5(4.7 \%)$ \\
Pulse rate $(\mathrm{N}=106)$ & $69(65.1 \%)$ & $5(4.7 \%)$ & $32(30.2 \%)$ \\
Respiratory rate & $36(34.0 \%)$ & $2(1.9 \%)$ & $68(64.2 \%)$ \\
$(\mathrm{N}=106)$ & & & \\
MAP $(\mathrm{N}=93)^{\mathrm{a}}$ & $43(46.2 \%)$ & $4(4.3 \%)$ & $46(49.5 \%)$ \\
Clinical signs & & & \\
Pupil character & Normal & Miotic & Mydriatic \\
$\mathrm{N}=106$ & $83(78.3)$ & $17(16.0 \%)$ & $5(4.7 \%)$ \\
Skin status & Normal & Dry & Sweaty \\
$\mathrm{N}=106$ & $78(73.6 \%)$ & $15(14.2 \%)$ & $13(12.3 \%)$ \\
Mental status & Normal & Altered & Agitated \\
$\mathrm{N}=106$ & $40(37.7 \%)$ & $66(62.3 \%)$ & $14(13.2 \%)$ \\
Gastrointestinal & Normal & Vomiting & Diarrhoea \\
$\mathrm{N}=106$ & $84(79.2 \%)$ & $14(13.2 \%)$ & $8(7.6 \%)$ \\
Genital urinary & Normal & Urine incontinence & \\
$\mathrm{N}=106$ & $95(89.6 \%)$ & $11(10.4 \%)$ & \\
Laboratory results & & & High \\
Random blood glucose & Normal & Low & $8(7.5 \%)$ \\
$\mathrm{N}=106$ & $92(86.8 \%)$ & $6(5.7 \%)$ & \\
\hline
\end{tabular}

${ }^{a}$ Mean arterial pressure

in adults $59(95.2 \%)$. Most common route of exposure was oral $(88$ [83.0\%]), followed by bites or stings by venomous animal/insects (11 [10.4\%]) (Table 5).

\section{Patient disposition and outcome}

Overall, most of the patients (98 [92.5\%]) were admitted to the hospital, 7 (6.6\%) were discharged home from the $\mathrm{ED}$, and one patient $(0.9 \%)$ died in the ED.

\section{Discussion}

Most of the patients in our study population were from Dar es Salaam city, reflecting similar patterns of referral to the MNH ED shown in previous studies [25]. We found that the majority of acute poisoning among patients presenting to our ED in Tanzania are males, with a 3:1 male to female ratio. These findings are similar to other studies done in Sub Saharan Africa, where acute poisoning is known to be common among young males [26]. In our study population, single, adult males with a limited level of education represented nearly one-third, which is consistent with a study by Ndosi et al. on fatal self-poisoning patients in Tanzania in which most of the patients were single, with a primary level of education [15]. However, contrary to observations made in other studies in similar settings $[15,26]$, over one-quarter of our patients had professional jobs (including nurse, 
Table 4 Suspected agents in all patients

\begin{tabular}{lr}
\hline Adult $\geq \mathbf{1 8}$ years & \\
\hline Toxic agent & $\mathbf{N}=\mathbf{8 4}$ \\
\hline Alcohol & $42(50.0 \%)$ \\
Mixture of different medications ${ }^{\mathrm{a}}$ & $12(14.3 \%)$ \\
Unknown & $8(9.5 \%)$ \\
Sedative or hypnotic agent & $7(8.3 \%)$ \\
Organophosphates & $7(8.3 \%)$ \\
Snake venom & $6(7.1 \%)$ \\
Rodenticide (rat poison) & $5(5.9 \%)$ \\
Anti-malarial (Artemisinin based combination) & $2(2.4 \%)$ \\
Tricyclic antidepressants & $2(2.4 \%)$ \\
Opioids & $2(2.4 \%)$ \\
\hline Children (<18 years) & \\
\hline Toxic agent & $\mathbf{N}=\mathbf{2 2}$ \\
\hline Snake venom & $6(27.3 \%)$ \\
Kerosene & $3(13.6 \%)$ \\
Rat poison & $3(13.6 \%)$ \\
Poly pharmacy & $2(9.1 \%)$ \\
Insecticide & $1(4.6 \%)$ \\
Acid & $1(4.6 \%)$ \\
Antimalarial & $1(4.6 \%)$ \\
Pesticide & $1(4.6 \%)$ \\
Tricyclic antidepressant & $1(4.6 \%)$ \\
Organophosphate & $1(4.6 \%)$ \\
\hline a &
\end{tabular}

a Includes: ethanol plus sedatives, ethanol plus rat poisoning, ethanol plus antimalarial and antibiotics, ethanol plus unknown agents

engineer, lawyer, businessman, and accountant), with most of these professionals presenting with intentional, recreational poisonings.
Contrary to observations made in most high-income countries [27, 28], alcohol taken for recreational purposes was found to be the most common toxic agent in our study population. These findings are different from earlier studies of poisoning done in Tanzania which showed that the most common substances used for fatal self-poisoning were antimalarials and pesticides. This difference may be due to our inclusion of all patients with suspected toxic exposure regardless of intentionality or suicidality, while both previous studies evaluated deceased patients or those with suicidal intention.

In evaluating children as a subset of our study population, we found that most common toxic exposure was snakebite, followed by accidental ingestion of kerosene or rodenticides. Contrary to findings of previous studies in similar settings [26] our findings of snakebites are interesting and suggests the need for a larger multicenter study which can lead to dedicated protocols of snakebite management. We were unable to identify the exact contents of the rodenticides but the most common products available in Tanzania include warfarins and related anticoagulants, zinc or aluminium phosphide, and organophophates. Despite having no reported deaths in the group of children who had rat poisoning, the diversity of the contents and serious potential toxicity continue to pose a significant challenge.

Nearly two-thirds of the toxic exposures occurred at home or in recreational areas, and over half of the toxic exposures were intentional. Intentional poisoning was five times more common in adults than in children. Most children had their toxic exposure at home, similar to other studies from sub-Saharan Africa [29], which have suggested that the culture of leaving children unattended is one important risk factor for domestic based accidents,

Table 5 Route of exposure and intent for all age groups

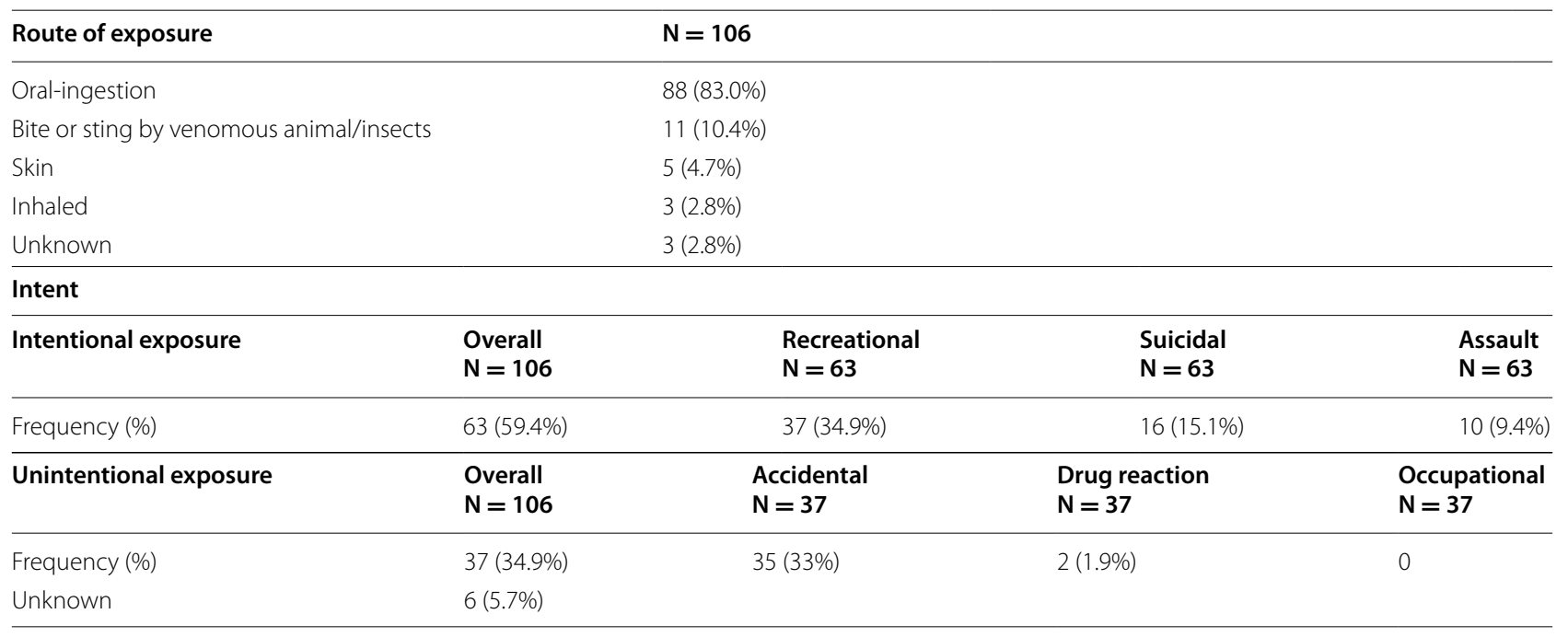


including poisoning $[29,30]$. Most of the intentional toxic exposures were due to recreational use of alcohol and only about a quarter were due to suicidal intention. Community based behavioral interventions [31] may be part of the solution to tackling acute alcohol poisoning incidents in Tanzania.

Clinical presentations of toxic exposures depend on the specific agent involved, the quantity absorbed, and the intent of exposure. The most common presenting clinical feature in our study population was altered mental status, which occurred in over $60 \%$ of patients. The differential diagnoses and management strategies of patients with altered mental status are widely variable [32], and health providers should always consider poisoning as a potential cause for altered mental status in patients presenting to acute intake areas in Tanzania.

Conservative management-including intravenous fluids and anti-pain-was the main stay of treatment for our patients, and over $90 \%$ of these patients were admitted to the hospital, three of who were intubated and admitted to intensive care units (ICU). In our setting mechanical ventilation and ICU care is significantly limited by availability of acute care beds and ventilator machines [33]. As such, we believe it is inappropriate to use ICU admission or intubation as markers of illness severity in this setting. Less than $10 \%$ of patients were discharged from the ED, half of who had a discharge diagnosis of alcohol intoxication. All patients who were discharged had stable vital signs; however, due to the nature and timing of the study, and the available resources, we were unable to follow up the discharged patients to investigate their outcomes after discharge. In this study, one patient died in the ED and the toxic substance that was involved was unknown and, as is common in our setting, no postmortem examination was performed.

\section{Limitations}

This was a single site descriptive study with a small sample size over a short period of time, at the ED of a large public tertiary referral centre, hence our results may not necessarily be generalizable to other EDs with different patient populations in difference settings. Furthermore, the potential for seasonal variations might not be ascertained within our findings. We relied on the treating doctors' clinical impression to identify eligible patients. These doctors may have variable ability to diagnose toxicological syndromes depending on their level of training and experience, and we have no laboratory analysis capabilities for toxicology screening. As such, we suspect that many toxicology patients pass through the MNH-EMD undiagnosed.

\section{Conclusions}

The majority of patients presenting to our ED with poisoning are adult male with intentional exposures, mostly after recreational use of alcohol. Substances used in adult suicidal ingestions included rodenticides, acid, antimalarials, organophosphates, tricyclic antidepressants, haloperidol and opioids. Substances involved in childhood poisonings included kerosene, rodenticides, antimalarials, antibiotics and ethanol. Future studies should focus on larger multicentre patient population and laboratory confirmation of the suspected poisoning agents.

\section{Abbreviations \\ ED: emergency department; CRF: case report form; WHO: World Health Organization; MNH: Muhimbili National Hospital; CBC: complete blood count; MUHAS: Muhimbili University of Health and Allied Sciences.}

\section{Authors' contributions}

GM, TR, HS contributed to the conception and design of the study, acquired, analyzed and interpreted the data, and drafted and revised the manuscript. SL, $J \mathrm{M}$, JS contributed to the design of the study, data acquisition and entry and also revised the manuscript. KO, VM, MR contributed to the conception and assisted in the initial design of the study and critically revised the manuscript. GM, HS, TR contributed to the conception and assisted in the initial design of the study, data interpretation and critically revised the manuscript, contributed to the conception and assisted in the initial design of the study, analyzed and interpreted the data and critically revised the manuscript. All authors read and approved the final manuscript.

\section{Author details \\ ${ }^{1}$ Emergency Medicine Department, Muhimbili University of Health and Allied Sciences, Dar es Salaam, P.O. Box 54235, Tanzania. ${ }^{2}$ Emergency Medicine Department, Muhimbili National Hospital, Dar es Salaam, Tanzania. ${ }^{3}$ Depart- ment of Emergency Medicine, University of California San Francisco, San Francisco, CA, USA. ${ }^{4}$ Department of Emergency Medicine, Carolinas Medical Center, Charlotte, NC, USA. ${ }^{5}$ Emergency and Trauma Care Lead, World Health Organisation, Geneva, Switzerland. ${ }^{6}$ California Poison Control System, Univer- sity of California, San Francisco, CA, USA.}

\section{Acknowledgements}

Thank you to Mr. Carlos Henriques and MNH-EMD staff for their tireless effort towards production of this manuscript.

\section{Competing interests}

The authors declare that they have no competing interests.

\section{Availability of data and materials}

The dataset supporting the conclusions of this article is available from the authors on request.

\section{Consent to publish}

Not applicable.

\section{Ethics approval and consent to participate}

The study protocol was reviewed and approved by the Institutional Review Board of the Muhimbili University of Health and Allied Sciences (MUHAS). The MUHAS Institutional Review Board approved the study protocol and all adult patients provided written informed consent, in person or through an eligible proxy. In children, the parent or guardian accompanying the child provided a written informed consent.

\section{Funding}

This was a non-funded project; the principal investigators used their own funds to support the data collection and logistics. 


\section{Publisher's Note}

Springer Nature remains neutral with regard to jurisdictional claims in published maps and institutional affiliations.

Received: 22 August 2016 Accepted: 8 September 2017 Published online: 16 September 2017

\section{References}

1. Z'gambo J, Siulapwa Y, Michelo C. Pattern of acute poisoning at two urban referral hospitals in Lusaka. Zambia. BMC Emerg Med. 2016;16:2. doi:10.1186/s12873-016-0068-3.

2. Kumar SV, Venkateswarlu B, Sasikala M, Kumar GV. A study on poisoning cases in a tertiary care hospital. J Nat Sci Biol Med. 2010;1(1):35-9.

3. Sahin S, Bora Carman K, Dinleyici EC. Acute poisoning in children; Data of a Pediatric Emergency Unit. Iran J Pediatr. 2011;21(4):479-84.

4. Ezeonwu B, Chima O, Oguonu T, Ikefuna A, Nwafor I. Morbidity and mortality pattern of childhood illnesses seen at the Children Emergency Unit of Federal Medical Center, Asaba, Nigeria. Ann Med Health Sci Res. 2014:4(Suppl 3):S239-44.

5. WHO Progress report. WHO. Towards universal access: scaling up priority HIV/AIDS interventions in the health sector-2013.

6. Abdollahi M, Jalali N, Sabzevari O, Hoseini R, Ghanea T. A retrospective study of poisoning in Tehran. J Toxicol Clin Toxicol. 1997:35(4):387-93.

7. Waugh J, Najafi J, Hawkins L, Hill SL, Eddleston M, Vale JA, et al. Epidemiology and clinical features of toxicity following recreational use of synthetic cannabinoid receptor agonists: a report from the United Kingdom national poisons information service. Clin Toxicol (Phila). 2016;19:1-7.

8. Bosak A, Brooks DE, Welch S, Padilla-Jones A, Gerkin RD. A retrospective review of 911 calls to a regional poison control center. J Family Med Prim Care. 2015:4(4):546-50.

9. Jaraczewska W, Kotwica M, Jarosz A, Kołaciński Z. Telephone toxicological information service on pesticide poisonings. Prz Lek. 1997;54(10):731-3.

10. Singh $O$, Javeri Y, Juneja D, Gupta M, Singh G, Dang R. Profile and outcome of patients with acute toxicity admitted in intensive care unit: experiences from a major corporate hospital in urban India. Indian J Anaesth. 2011;55(4):370-4.

11. Bronstein AC, Spyker DA, Cantilena LR Jr, Green JL, Rumack BH, Dart RC. 2010 annual report of the american association of poison control centers' national poison data system (NPDS): 28th annual report. Clin Toxicol (Phila). 2011;49(10):910-41.

12. Lang T, Thuo $N$, Akech S. Accidental paraffin poisoning in Kenyan children. Trop Med Int Health. 2008;13(6):845-7.

13. Nhachi CF, Kasilo OM. Household chemicals poisoning admissions in Zimbabwe's main urban centres. Hum Exp Toxicol. 1994;13(2):69-72.

14. Balme KH, Roberts JC, Glasstone M, Curling L, Mann MD. The changing trends of childhood poisoning at a tertiary children's hospital in South Africa. S Afr Med J. 2012;102(3 Pt 1):142-6.

15. Ndosi NK, Mbonde MP, Lyamuya E. Profile of suicide in Dar es Salaam. East Afr Med J. 2004;81(4):207-11.
16. Mlingi NLV, Nkya S, Tatala SR, Rashid S, Bradbury JH. Recurrence of konzo in southern Tanzania: rehabilitation and prevention using the wetting method. Food Chem Toxicol. 2011;49(3):673-7.

17. Bose-O'Reilly S, Drasch G, Beinhoff C, Tesha A, Drasch K, Roider G, et al. Health assessment of artisanal gold miners in Tanzania. Sci Total Environ. 2010;408(4):796-805

18. Spiegel SJ. Occupational health, mercury exposure, and environmental justice: learning from experiences in Tanzania. Am J Public Health. 2009;99(Suppl 3):S550-8.

19. Azayo MM, Manji K, Kalokola F. Lead levels in women at delivery at the Muhimbili National Hospital: a public health problem. J Trop Pediatr. 2009;55(2):138-9.

20. Boyle JS, Bechtel LK, Holstege CP. Management of the critically poisoned patient. Scand J Trauma Resusc Emerg Med. 2009;29(17):29.

21. Ramesha KN, Rao KBH, Kumar GS. Pattern and outcome of acute poisoning cases in a tertiary care hospital in Karnataka, India. Indian J Crit Care Med. 2009;13(3):152-5.

22. Spiller HA, Griffith JRK. The value and evolving role of the US Poison Control Center System. Public Health Rep. 2009;124(3):359-63.

23. Morton WS. Hawaii poison center. Forty years of saving lives and health costs. Hawaii Med J. 1998;57(3):440-2.

24. Reynolds T, Sawe HR, Lobue N, Mwafongo V. Most frequent adult and pediatric diagnoses among 60,000 patients seen in a new urban emergency department in Dar Es Salaam, Tanzania. Ann Emerg Med. 2012;60(4):S39.

25. Microsoft Word_EAJPH Volume 5 Number 1 April 2008_Corrected.docpdf [Internet]. Available from: http://www.bioline.org.br/pdf?lp08002. Accessed Apr 222016.

26. Mgaya E, Kazaura MR, Outwater A, Kinabo L. Suicide in the Dar es Salaam region, Tanzania, 2005. J Forensic Leg Med. 2008;15(3):172-6.

27. Yang CC, Wu JF, Ong HC, Hung SC, Kuo YP, Sa CH, et al. Taiwan National Poison Center: epidemiologic data 1985-1993. J Toxicol Clin Toxicol. 1996:34(6):651-63.

28. Hoppe-Roberts JM, Lloyd LM, Chyka PA. Poisoning mortality in the United States: comparison of national mortality statistics and poison control center reports. Ann Emerg Med. 2000;35(5):440-8.

29. WHO. World Report on child injury prevention [Internet]. WHO. Available from: http://www.who.int/violence_injury_prevention/child/injury/ world_report/report/en/. Accessed Apr 242016.

30. Parbhoo A, Louw QA, Grimmer-Somers K. A profile of hospital-admitted paediatric burns patients in South Africa. BMC Res Notes. 2010;11(3):165.

31. Papas RK, Sidle JE, Martino S, Baliddawa JB, Songole R, Omolo OE, et al. Systematic cultural adaptation of cognitive-behavioral therapy to reduce alcohol use among HIV-infected outpatients in western Kenya. AIDS Behav. 2010;14(3):669-78.

32. Odiari EA, Sekhon N, Han JY, David EH. Stabilizing and managing patients with altered mental status and delirium. Emerg Med Clin North Am. 2015;33(4):753-64

33. Sawe HR, Mfinanga JA, Lidenge SJ, Mpondo BC, Msangi S, Lugazia E, et al. Disease patterns and clinical outcomes of patients admitted in intensive care units of tertiary referral hospitals of Tanzania. BMC Int Health Hum Rights. 2014;23(14):26.

\section{Submit your next manuscript to BioMed Central and we will help you at every step:}

- We accept pre-submission inquiries

- Our selector tool helps you to find the most relevant journal

- We provide round the clock customer support

- Convenient online submission

- Thorough peer review

- Inclusion in PubMed and all major indexing services

- Maximum visibility for your research

Submit your manuscript at www.biomedcentral.com/submit 